\title{
3D Sparse Signal Recovery via 3D Orthogonal Matching Pursuit ${ }^{\text {th }}$
}

\author{
Yingqiu Huo ${ }^{\mathrm{a}}$, Yong Fang, ${ }^{*}$, Lei Huang ${ }^{\mathrm{b}}$ \\ ${ }^{a}$ College of Mechanical and Electronic Engineering, Northwest A\&3F University, Shaanxi \\ Yangling 712100, China \\ ${ }^{b}$ Department of Electronic and Information Engineering, Shenzhen Graduate School, \\ Harbin Institute of Technology, Guangdong Shenzhen 518055, China
}

\section{Abstract}

Though many three-dimensional (3D) compressive sensing schemes have been proposed, recovery algorithms in most of these schemes are designed for 1D or 2D signals, which cause some serious drawbacks, e.g., huge memory usage, and high decoder complexity. This paper proposes a 3D separable operator (3DSO) which is able to completely exploit the spatial and spectral correlation to sparsify and samples the 3D signal in three dimensions. A 3D orthogonal matching pursuit (3D-OMP) algorithm is then employed to recover the $3 \mathrm{D}$ sparse signal, which is able to reduce the computational complexity of the decoder significantly with guaranteed accuracy. In the proposed algorithm, we represent each 3D signal as a weighted sum of 3D atoms, which allow us to sample the 3D signal with 3D separable sensing operator. Then the best matched atoms are selected to construct the 3D support set, and the $3 \mathrm{D}$ signal is optimally recovered from the 3D support set in the sense

\footnotetext{
Supported by National Science Foundation of China

* Corresponding author

Email addresses: fallying@gmail.com (Yingqiu Huo), yfang79@gmail.com (Yong Fang )
}

Preprint submitted to Journal of Systems Architecture

October 5, 2015

(C) 2015. This manuscript version is made available under the Elsevier user license http://www.elsevier.com/open-access/userlicense/1.0/ 
of the least squares. Experimental results show that the 3D-OMP approach achieves higher recovery quality but requires less computational time than the Kronecker Compressive Sensing (KCS) scheme.

Key words:

Compressive sensing, 3D sparse signal, 3D separable operator, 3D separable sampling, 3D orthogonal matching pursuit.

\section{Introduction}

Assume that $\boldsymbol{x} \in \mathbb{R}^{n}$ is a one-dimensional (1D) signal, which is sparse under a certain orthogonal transform basis $\Psi \in \mathbb{R}^{n \times n}$, where $\mathbb{R}$ is the set of real numbers, so that $\boldsymbol{x}=\boldsymbol{\Psi} \boldsymbol{z}$ and there are only $k \ll n$ spikes (nonzero entries) in $\boldsymbol{z}$. The measurement vector $\boldsymbol{y} \in \mathbb{R}^{m}$ can be expressed as $\boldsymbol{y}=$ $\mathbf{A} \boldsymbol{z} \in \mathbb{R}^{m}$, where $\mathbf{A}=\boldsymbol{\Phi} \boldsymbol{\Psi} \in \mathbb{R}^{m \times n}(m<n)$ and $\boldsymbol{\Phi} \in \mathbb{R}^{m \times n}$ is the sampling matrix. It is proved in [2, 3, 4] that when $\boldsymbol{\Phi}$ and $\boldsymbol{\Psi}$ are incoherent, and $\boldsymbol{\Phi}$ obeys the restricted isometry property (RIP) with a constant parameter, the sparse vector $\boldsymbol{z}$ can be recovered from $\boldsymbol{y}$ by solving the $\ell_{1}$-norm optimization problem:

$$
\arg \min _{\boldsymbol{z} \in \mathbb{R}^{m}}\|\boldsymbol{z}\|_{1} \quad \text { s.t. } \quad \boldsymbol{y}=\boldsymbol{\Phi} \boldsymbol{\Psi} \boldsymbol{z}=\mathbf{A} \boldsymbol{z} .
$$

Many recovery algorithms have been proposed in the literature, e.g., matching pursuit(MP) [9], orthogonal matching pursuit (OMP) [8, 9], regularized orthogonal pursuit (ROMP) [5], compressive sampling matching pursuit (CoSaMP) [6], and subspace pursuit (SP) [7]. However, most of these algorithms are originally designed for 1D signal recovery. For two-dimensional (2D) signals, e.g., the image and video signals, a straightforward approach of $2 \mathrm{D}$ compressive sensing (CS) is to stretch a matrix into a vector, which 
augments the length of the signal, and increases exponentially the computational complexity and memory usage at both the encoder and decoder. An alternative approach is to sample the rows and columns of the 2D signal, respectively, which considerably reduces the encoding complexity [10]. However, the decoding complexity is still very high. The 2D-OMP provides a good solution to this problem, which reduces the recovery complexity and memory usage significantly [11].

To handle three-dimensional (3D) signals, e.g., the hyper-spectral image (HSI) and video signals, a number of schemes have been devised. An adaptive method has been suggested in [12], in which each video frame is split into non-overlapping blocks with equal size, and the sparse blocks are reconstructed by the OMP while the remaining blocks are sampled fully. The video compressive sensing (VCS) algorithm [13] senses the reference frames of a video with full sampling while senses other frames by the standard CS. The distributed compressed video sensing (DCVS) approach first decodes the key frames and then recovers other frames by exploiting the inter-frame sparsity model and the sparse recovery algorithm with side information [14]. The joint method [15] recovers the HSI by exploiting the low-rank and jointsparse properties. The 3D-CS methods [16] recovers a video by using inexact augmented Lagrangian multipliers (ALM) and alternating direction method (ADM). Three reconstruction methodologies have been developed in [17] for confocal microscopy based on the framework of CS. An augmented Lagrangian algorithm has been suggested in [18] for solving the unmixing model to reconstruct signals. Moreover, the KCS scheme proposes to use the Kronecker product matrix as the sampling matrix and sparsifying base 
to handle the 3D signal [1]. However, most of the recovery algorithms in these schemes are only focused on 1D or 2D signals recovery. To the best of our knowledge, there is no work which represents the $3 \mathrm{D}$ sparse signal as a weighted sum of certain 3D atoms and recovers it from the 3D support set directly.

This paper proposes the 3DSO scheme which is consist of the 3D separable sampling (3DSS) and 3D sparsifying transform (3DST). The transform matrix $\boldsymbol{\Psi}$ and sampling matrix $\boldsymbol{\Phi}$ are adopted to sparsify and sample the 3D signal along three dimensions which are termed the 3DSS and 3DST, respectively. Furthermore, the 3D-OMP recovery algorithm is devised, in which the 3D signal can be decomposed into certain 3D atoms. We search for the best matched $3 \mathrm{D}$ atom by using the $3 \mathrm{D}$ projection to construct the $3 \mathrm{D}$ support set, then optimally recover the 3D sparse signal from the 3D support set in the sense of the least square. The simulation results indicate that the 3D-OMP is superior to the KCS.

The rest of this paper is organized as follows. Section 2 provides the 3DSO. Section 3 introduces the 3D-OMP algorithm. Section 4 addresses the complexity and memory usage of the 3D-OMP. Section 5 presents results. Finally, Section [6] concludes this paper.

\section{3D Separable Operator}

Let the tensor $\mathcal{X} \in \mathbb{R}^{n \times n \times n}$ be a 3D signal. The 3DSO uses the separable operators $\boldsymbol{\Phi}_{x}, \boldsymbol{\Phi}_{y}, \boldsymbol{\Phi}_{z} \in \mathbb{R}^{m \times n}$ to sample the row, column, and vertical dimension of $\mathcal{X}$, respectively. It can be obtained that $\mathcal{Y}=\mathcal{X} \times{ }_{1} \Phi_{x} \times_{2} \Phi_{y} \times_{3} \boldsymbol{\Phi}_{z} \in$ $\mathbb{R}^{m \times m \times m}$, where $\times_{1}, \times_{2}$, and $\times_{3}$ denote the $n$-mode product of a tensor with 
a matrix on the row, column, and vertical dimension [20]. Let us define three sparsifying transform bases: $\boldsymbol{\Psi}_{x}, \boldsymbol{\Psi}_{y}, \boldsymbol{\Psi}_{z} \in \mathbb{R}^{n \times n}$. So $\mathcal{X}$ can be written as $\mathcal{Z} \times{ }_{1} \boldsymbol{\Psi}_{x} \times{ }_{2} \boldsymbol{\Psi}_{y} \times{ }_{3} \boldsymbol{\Psi}_{z}$, where the sparsity base holds for most of popular transform matrices, e.g., the wavelet transform, discrete cosine transform (DCT), and Fourier transform, etc. The convex optimization problem of minimizing $\ell_{1}$-norm is:

$$
\arg \min _{\mathcal{Z} \in \mathbb{R}^{n \times n \times n}}\|\mathcal{Z}\|_{1} \text { s.t. } \mathcal{Y}=\left(\mathcal{Z} \times{ }_{1} \Psi_{x} \times_{2} \boldsymbol{\Psi}_{y} \times{ }_{3} \boldsymbol{\Psi}_{z}\right) \times_{1} \boldsymbol{\Phi}_{x} \times_{2} \boldsymbol{\Phi}_{y} \times_{3} \boldsymbol{\Phi}_{z}
$$

Let us define $\boldsymbol{z}=\operatorname{vec}(\operatorname{vec}(\mathcal{Z})) \in \mathbb{R}^{n^{3}}$ and $\boldsymbol{y}=\operatorname{vec}(\operatorname{vec}(\mathcal{Y})) \in \mathbb{R}^{m^{3}}$, which denote the column-section-ordered vectorization of the $3 \mathrm{D}$ matrix $\mathcal{Z}$ and $\mathcal{Y}$, respectively. In addition, $\operatorname{vec}(\mathcal{Z}) \in \mathbb{R}^{n^{2} \times n}$ denotes the section-ordered vectorization of the $3 \mathrm{D}$ matrix $\mathcal{Z}$. Then we obtain:

$$
\begin{aligned}
\boldsymbol{y} & =\operatorname{vec}\left\{\mathcal{Z} \times_{1}\left(\boldsymbol{\Phi}_{x} \boldsymbol{\Psi}_{x}\right) \times_{2}\left(\boldsymbol{\Phi}_{y} \boldsymbol{\Psi}_{y}\right) \times_{3}\left(\boldsymbol{\Phi}_{z} \boldsymbol{\Psi}_{z}\right)\right\} \\
& =\left(\boldsymbol{\Phi}_{z} \otimes \boldsymbol{\Phi}_{y} \otimes \boldsymbol{\Phi}_{x}\right)\left(\boldsymbol{\Psi}_{z} \otimes \boldsymbol{\Psi}_{y} \otimes \boldsymbol{\Psi}_{x}\right) \boldsymbol{z}
\end{aligned}
$$

Thus (2) can be rewritten as:

$$
\arg \min _{\boldsymbol{z} \in \mathbb{R}^{n^{3}}}\|\boldsymbol{z}\|_{1} \text { s.t. } \boldsymbol{y}=\left(\boldsymbol{\Phi}_{z} \otimes \boldsymbol{\Phi}_{y} \otimes \boldsymbol{\Phi}_{x}\right)\left(\boldsymbol{\Psi}_{z} \otimes \boldsymbol{\Psi}_{y} \otimes \boldsymbol{\Psi}_{x}\right) \boldsymbol{z},
$$

which indicates that the $3 \mathrm{DSO}$ is a special case of the $\ell_{1}$-norm minimization problem where the sample and sparsifying matrices are the Kronecker product of three sample matrices and three sparsifying matrices, respectively.

In contrast to the standard CS which samples the vectorized signal $\boldsymbol{z} \in$ $\mathbb{R}^{n^{3}}$ of the $3 \mathrm{D}$ signal with the sampling matrix $\mathbf{A}_{1 D} \in \mathbb{R}^{m^{3} \times n^{3}}(m<n)$, the 3DSS method samples $\mathbf{Z}$ with the sampling matrix $\mathbf{A}_{3 D} \in \mathbb{R}^{m \times n}$ in the $x, y$, and $z$ dimensions, respectively, and hence reduces the complexity significantly while achieving the identical effect. The 3D signal can also be handled 
through 2D-CS schemes, e.g., the 2D-OMP[11], in which the $3 \mathrm{D}$ signal is regarded as a collection of $2 \mathrm{D}$ images. This results in the same sampling matrix $\boldsymbol{\Phi}_{2 D} \in \mathbb{R}^{m \times n}$ and sparsifying transform matrix $\boldsymbol{\Psi}_{2 D} \in \mathbb{R}^{n \times n}$ applied to each 2D image, which neglects the remarkable high correlation in spectral domain. However, the 3DSO makes full use of the high spectral correlation to sparsify and sample each 3D signal in all dimensions, respectively. Thus it can obtain higher recovered quality and elapse shorter time.

\subsection{Mutual Coherence}

Let $s=m^{3}$, which denotes the number of samples, and $w=n^{3}$, which denotes the pixel number of a 3D signal. As shown in [10], $s$ is proportional to $\mu^{2}$, i.e.

$$
s \geq c \cdot \mu^{2}\left\{\left(\boldsymbol{\Phi}_{z} \otimes \boldsymbol{\Phi}_{y} \otimes \boldsymbol{\Phi}_{x}\right),\left(\boldsymbol{\Psi}_{z} \otimes \boldsymbol{\Psi}_{y} \otimes \boldsymbol{\Psi}_{x}\right)\right\} \cdot k \cdot \log (w),
$$

where $c$ is a small positive constant, and $\mu$ is the mutual coherence [1] which is defined as:

$$
\begin{aligned}
& \mu\left\{\left(\boldsymbol{\Phi}_{z} \otimes \boldsymbol{\Phi}_{y} \otimes \boldsymbol{\Phi}_{x}\right),\left(\boldsymbol{\Psi}_{z} \otimes \boldsymbol{\Psi}_{y} \otimes \boldsymbol{\Psi}_{x}\right)\right\} \\
= & \sqrt{w} \cdot \max _{i, o}\left|\left\langle\left(\boldsymbol{\Phi}_{z} \otimes \boldsymbol{\Phi}_{y} \otimes \boldsymbol{\Phi}_{x}\right)_{i},\left(\boldsymbol{\Psi}_{z} \otimes \boldsymbol{\Psi}_{y} \otimes \boldsymbol{\Psi}_{x}\right)_{o}\right\rangle\right| \\
= & \sqrt{n^{3}} \cdot \max _{i, j, k, o, p, q}\left|\left\langle\boldsymbol{\phi}_{i}^{z} \otimes \boldsymbol{\phi}_{j}^{y} \otimes \boldsymbol{\phi}_{k}^{x}, \boldsymbol{\psi}_{o}^{z} \otimes \boldsymbol{\psi}_{p}^{y} \otimes \boldsymbol{\psi}_{q}^{x}\right\rangle\right| \\
= & (\sqrt{n})^{3} \cdot \max _{i, j, k, o, p, q}\left|\left\langle\boldsymbol{\phi}_{i}^{z}, \boldsymbol{\psi}_{o}^{z}\right\rangle\left\langle\boldsymbol{\phi}_{j}^{y}, \boldsymbol{\psi}_{p}^{y}\right\rangle\left\langle\boldsymbol{\phi}_{k}^{x}, \boldsymbol{\psi}_{q}^{x}\right\rangle\right| \\
= & \sqrt{n} \max _{i, o}\left|\left\langle\boldsymbol{\phi}_{i}^{z}, \boldsymbol{\psi}_{o}^{z}\right\rangle\right| \cdot \sqrt{n} \max _{j, p}\left|\left\langle\boldsymbol{\phi}_{j}^{y}, \boldsymbol{\psi}_{p}^{y}\right\rangle\right| \cdot \sqrt{n} \max _{k, q}\left|\left\langle\boldsymbol{\phi}_{k}^{x}, \boldsymbol{\psi}_{q}^{x}\right\rangle\right| \\
= & \mu\left(\boldsymbol{\Phi}_{x}, \boldsymbol{\Psi}_{x}\right) \mu\left(\boldsymbol{\Phi}_{y}, \boldsymbol{\Psi}_{y}\right) \mu\left(\boldsymbol{\Phi}_{z}, \boldsymbol{\Psi}_{z}\right) \\
\geq & \min _{i=x, y, z}\left(\mu^{3}\left(\boldsymbol{\phi}_{i}, \boldsymbol{\psi}_{i}\right)\right) .
\end{aligned}
$$

Here $\boldsymbol{\phi}_{i}$, and $\boldsymbol{\psi}_{j} \in \mathbb{R}^{m}$ denote the column vectors of $\boldsymbol{\Phi}$ and $\boldsymbol{\Psi}$, respectively. Equation (6) implies that we can find a sampling matrix $\boldsymbol{\Phi}$ and a sparsity 
base $\Psi$ to guarantee the smallest mutual coherence among them. When $\boldsymbol{\Psi}_{x}=\boldsymbol{\Psi}_{y}=\boldsymbol{\Psi}_{z}=\boldsymbol{\Psi} \in \mathbb{R}^{n \times n}$, and $\boldsymbol{\Phi}_{x}=\boldsymbol{\Phi}_{y}=\boldsymbol{\Phi}_{z}=\boldsymbol{\Phi} \in \mathbb{R}^{m \times n}$, equation (2) can be rewritten:

$$
\mathcal{Y}=\mathcal{Z} \times{ }_{1} \mathbf{A} \times{ }_{2} \mathbf{A} \times{ }_{3} \mathbf{A} \in \mathbb{R}^{m \times m \times m}
$$

where $\mathbf{A}=\boldsymbol{\Phi} \Psi \in \mathbb{R}^{m \times n}$, and the mutual coherence is the smallest one.

\subsection{Restricted Isometry Constant}

The Restricted Isometry Constant (RIC) of the Kronecker product sampling matrix $\Theta$ is related to the RICs of all submatrices of $\Theta$. From 4 , it is obtained that

$$
\begin{aligned}
& \left(\boldsymbol{\Phi}_{z} \otimes \boldsymbol{\Phi}_{y} \otimes \boldsymbol{\Phi}_{x}\right)\left(\boldsymbol{\Psi}_{z} \otimes \boldsymbol{\Psi}_{y} \otimes \boldsymbol{\Psi}_{x}\right) \\
= & \left(\boldsymbol{\Phi}_{z} \boldsymbol{\Psi}_{z}\right) \otimes\left(\boldsymbol{\Phi}_{y} \boldsymbol{\Psi}_{y}\right) \otimes\left(\boldsymbol{\Phi}_{x} \boldsymbol{\Psi}_{x}\right) \\
= & \mathbf{A}_{z} \otimes \mathbf{A}_{y} \otimes \mathbf{A}_{x}
\end{aligned}
$$

Let $\boldsymbol{\Theta}=\mathbf{A}_{z} \otimes \mathbf{A}_{y} \otimes \mathbf{A}_{x}$, and $\boldsymbol{\Theta}_{\Gamma}$ denotes the $k$-column submatrix of $\boldsymbol{\Theta}$ where $\Gamma$ is the index set with $|\Gamma| \leq k$. Meanwhile, let $\lambda_{\min }\left(\boldsymbol{\Theta}_{\Gamma}^{\mathrm{T}} \boldsymbol{\Theta}_{\Gamma}\right)$ and $\lambda_{\max }\left(\boldsymbol{\Theta}_{\Gamma}^{\mathrm{T}} \boldsymbol{\Theta}_{\Gamma}\right)$ be the minimum and maximum eigenvalues of the matrix $\boldsymbol{\Theta}_{\Gamma}$. Then

$$
1-\delta_{k}(\boldsymbol{\Theta}) \leq \lambda_{\min }\left(\boldsymbol{\Theta}_{\Gamma}^{\mathrm{T}} \boldsymbol{\Theta}_{\Gamma}\right) \leq \frac{\left\|\boldsymbol{\Theta}_{\Gamma} z\right\|_{2}^{2}}{\|z\|_{2}^{2}} \leq \lambda_{\max }\left(\boldsymbol{\Theta}_{\Gamma}^{\mathrm{T}} \boldsymbol{\Theta}_{\Gamma}\right) \leq 1+\delta_{k}(\boldsymbol{\Theta})
$$

holds for all $k$-sparse vector $z \in \mathbb{R}^{n^{3}}$ where $\delta_{k}(\boldsymbol{\Theta}) \geq 0$ is the RIC of order $k$.

Because

$$
\left\{\begin{array}{l}
\lambda_{\min }\left(\boldsymbol{\Theta}_{\Gamma}^{\mathrm{T}} \boldsymbol{\Theta}_{\Gamma}\right)=\left|\sigma_{\min }\left(\boldsymbol{\Theta}_{\Gamma}\right)\right|^{2} \\
\lambda_{\max }\left(\boldsymbol{\Theta}_{\Gamma}^{\mathrm{T}} \boldsymbol{\Theta}_{\Gamma}\right)=\left|\sigma_{\max }\left(\boldsymbol{\Theta}_{\Gamma}\right)\right|^{2}
\end{array},\right.
$$


we have

$$
1-\delta_{k}(\boldsymbol{\Theta}) \leq\left|\sigma_{\min }\left(\boldsymbol{\Theta}_{\Gamma}\right)\right|^{2} \leq\left|\sigma_{\max }\left(\boldsymbol{\Theta}_{\Gamma}\right)\right|^{2} \leq 1+\delta_{k}(\boldsymbol{\Theta})
$$

Since $\boldsymbol{\Theta}_{\Gamma}$ is a submatrix of $\boldsymbol{\Theta}$, its column vector satisfies $\theta_{r}=a_{u}^{z} \otimes a_{v}^{y} \otimes a_{w}^{x}$, where $r \in \Gamma, u \in \Gamma_{z}, v \in \Gamma_{y}, w \in \Gamma_{x}$. It is easy to obtain that $\boldsymbol{\Theta}_{\Gamma}$ is the submatrix of $\mathbf{A}_{\Gamma_{y}}^{z} \otimes \mathbf{A}_{\Gamma_{y}}^{y} \otimes \mathbf{A}_{\Gamma_{x}}^{x}$. According to the interlacing theorem for singular values [19], the singular values of a submatrix is within the range of minimum and maximum singular values of the original matrix. Hence,

$\sigma_{\min }\left(\mathbf{A}_{\Gamma_{z}}^{z} \otimes \mathbf{A}_{\Gamma_{y}}^{y} \otimes \mathbf{A}_{\Gamma_{x}}^{x}\right) \leq \sigma_{\min }\left(\boldsymbol{\Theta}_{\Gamma}\right) \leq \sigma_{\max }\left(\boldsymbol{\Theta}_{\Gamma}\right) \leq \sigma_{\max }\left(\mathbf{A}_{\Gamma_{z}}^{z} \otimes \mathbf{A}_{\Gamma_{y}}^{y} \otimes \mathbf{A}_{\Gamma_{x}}^{x}\right)$.

Using the fact that

$$
\left\{\begin{array}{c}
\sigma_{\min }\left(\mathbf{A}_{\Gamma_{z}}^{z} \otimes \mathbf{A}_{\Gamma_{y}}^{y} \otimes \mathbf{A}_{\Gamma_{x}}^{x}\right)=\sigma_{\min }\left(\mathbf{A}_{\Gamma_{z}}^{z}\right) \sigma_{\min }\left(\mathbf{A}_{\Gamma_{y}}^{y}\right) \sigma_{\min }\left(\mathbf{A}_{\Gamma_{x}}^{x}\right) \\
\sigma_{\max }\left(\mathbf{A}_{\Gamma_{z}}^{z} \otimes \mathbf{A}_{\Gamma_{y}}^{y} \otimes \mathbf{A}_{\Gamma_{x}}^{x}\right)=\sigma_{\max }\left(\mathbf{A}_{\Gamma_{z}}^{z}\right) \sigma_{\max }\left(\mathbf{A}_{\Gamma_{y}}^{y}\right) \sigma_{\max }\left(\mathbf{A}_{\Gamma_{x}}^{x}\right)
\end{array},\right.
$$

and

$$
\left\{\begin{array}{l}
1-\delta_{k}\left(\mathbf{A}_{z}\right) \leq\left|\sigma_{\min }\left(\mathbf{A}_{\Gamma_{z}}^{z}\right)\right|^{2} \leq\left|\sigma_{\max }\left(\mathbf{A}_{\Gamma_{z}}^{z}\right)\right|^{2} \leq 1+\delta_{k}\left(\mathbf{A}_{z}\right) \\
1-\delta_{k}\left(\mathbf{A}_{y}\right) \leq\left|\sigma_{\min }\left(\mathbf{A}_{\Gamma_{y}}^{y}\right)\right|^{2} \leq\left|\sigma_{\max }\left(\mathbf{A}_{\Gamma_{y}}^{y}\right)\right|^{2} \leq 1+\delta_{k}\left(\mathbf{A}_{y}\right) \\
1-\delta_{k}\left(\mathbf{A}_{x}\right) \leq\left|\sigma_{\min }\left(\mathbf{A}_{\Gamma_{x}}^{x}\right)\right|^{2} \leq\left|\sigma_{\max }\left(\mathbf{A}_{\Gamma_{x}}^{x}\right)\right|^{2} \leq 1+\delta_{k}\left(\mathbf{A}_{x}\right)
\end{array}\right.
$$

where $\delta_{k}\left(\mathbf{A}_{z}\right), \delta_{k}\left(\mathbf{A}_{y}\right)$, and $\delta_{k}\left(\mathbf{A}_{x}\right)$ are the restricted isometry constant of $\mathbf{A}_{z}$, $\mathbf{A}_{y}$, and $\mathbf{A}_{x}$, respectively. Then we can get

$$
\prod_{i=x, y, z} 1-\delta_{k}\left(\mathbf{A}_{i}\right) \leq\left|\sigma_{\min }\left(\boldsymbol{\Theta}_{\Gamma}\right)\right|^{2} \leq\left|\sigma_{\max }\left(\boldsymbol{\Theta}_{\Gamma}\right)\right|^{2} \leq \prod_{i=x, y, z} 1+\delta_{k}\left(\mathbf{A}_{i}\right)
$$

Hence,

$$
\begin{aligned}
\delta_{k}(\boldsymbol{\Theta}) & =\delta_{k}\left(\mathbf{A}_{z} \otimes \mathbf{A}_{y} \otimes \mathbf{A}_{x}\right) \\
& =\max \left\{1-\prod_{i=x, y, z} 1-\delta_{k}\left(\mathbf{A}_{i}\right), \prod_{i=x, y, z}\left(1+\delta_{k}\left(\mathbf{A}_{i}\right)\right)-1\right\}
\end{aligned}
$$


When $\mathbf{A}_{z}=\mathbf{A}_{y}=\mathbf{A}_{x}=\mathbf{A}$, the singular value of $\boldsymbol{\Theta}$ is $\max \left\{1-\prod 1-\right.$ $\left.\delta_{k}(\mathbf{A}), \prod\left(1+\delta_{k}(\mathbf{A})\right)-1\right\}$.

\section{3D-OMP Algorithm}

In this section, we first define the 3D atom, 3D dictionary, and the projection of a 3D sample matrix onto a 3D atom. Then we give the detailed derivation of the 3D-OMP and analyze its complexity. Algorithm 1 1 summarizes the main steps of the 3D-OMP. We define the set $(\boldsymbol{i}, \boldsymbol{j}, \boldsymbol{k})$ to record the coordinates of those selected atoms and $\mathcal{R} \in \mathbb{R}^{m \times m \times m}$ to hold the residue. At each iteration the recovery algorithm first searches for the best matched atom in the dictionary to construct the support set of $3 \mathrm{D}$ atoms, and then reconstructs the 3D sparse signal on the basis of the support set via the least square.

\section{1. $3 D$ Atom and Dictionary}

The 3D-OMP algorithm takes the sample matrix $\mathcal{Y} \in \mathbb{R}^{m \times m \times m}$ as the weighted sum of $n^{3}$ 3D atoms, each atom is an $m \times m \times m$ matrix. Therefore, the dictionary contains $n^{3} 3 \mathrm{D}$ atoms. We denote the $(i, j, k)$-th 3D atom by $\mathcal{C}_{i, j, k}$, where $1 \leq i, j, k \leq n$. Let $\mathbf{A}=\left(\boldsymbol{a}_{1}, \cdots, \boldsymbol{a}_{n}\right)$, where $\boldsymbol{a}_{i}$ is the $i$-th column of $\mathbf{A}$. Then $\mathcal{C}_{i, j, k}$ is the outer product of $\boldsymbol{a}_{i}, \boldsymbol{a}_{j}$ and $\boldsymbol{a}_{k}$, i.e.,

$$
\mathcal{C}_{i, j, k}=\boldsymbol{a}_{i} \otimes \boldsymbol{a}_{j}^{\mathrm{T}} \otimes \boldsymbol{a}_{k}^{\mathrm{T}},
$$

and the structure of the 3D atom is plotted in Fig. 1.

Let $\Omega=\left(\mathcal{C}_{1,1,1}, \cdots, \mathcal{C}_{2,3,4}, \cdots, \mathcal{C}_{i, j, k}\right)$. We call $\Omega$ the $3 \mathrm{D}$ dictionary. Then it is obtained that:

$$
\mathcal{Y}=\sum_{i=1}^{n} \sum_{j=1}^{n} \sum_{k=1}^{n} z_{i, j, k} \mathcal{C}_{i, j, k}
$$




\begin{tabular}{l}
\hline Algorithm 1: 3D CS implemented by 3D-OMP \\
\hline Input:
\end{tabular}

- $\mathbf{A} \in \mathbb{R}^{m \times n}$ : sampling matrix

- $\mathcal{Y} \in \mathbb{R}^{m \times m \times m}:$ sample matrix

- $k$ : sparse level

\section{Output:}

- $\tilde{\mathcal{Z}} \in \mathbb{R}^{n \times n \times n}$ : reconstruction of the ideal signal $\mathbf{Z}$

\section{Variable:}

- $\mathcal{R} \in \mathbb{R}^{m \times m \times m}:$ residual

- $(\boldsymbol{i}, \boldsymbol{j}, \boldsymbol{k})$ : set of the coordinates of atoms that are allowed to be selected in the future, $\boldsymbol{i}$ stands for row indices, $\boldsymbol{j}$ stands for column indices, and $\boldsymbol{k}$ stands for the vertical indices

\section{Initialization:}

\section{- $\mathcal{R} \leftarrow \mathcal{Y}$}

- $(\boldsymbol{i}, \boldsymbol{j}, \boldsymbol{k}) \leftarrow\{(1,1,1),(1,1,2), \cdots,(n, n, n)\}$

for $t \leftarrow 1$ to $k$ do

$$
\begin{aligned}
& \left(i_{t}, j_{t}, k_{t}\right) \leftarrow \arg \max _{\left(i^{\prime}, j^{\prime}, k^{\prime}\right) \in(i, j, k)} \frac{\left|\left\langle\mathcal{R}, \mathcal{C}_{i^{\prime}, j^{\prime}, k^{\prime}}\right\rangle\right|}{\left\|\mathcal{c}_{i^{\prime}, j^{\prime}, k^{\prime}}\right\|_{2}} \\
& (\boldsymbol{i}, \boldsymbol{j}, \boldsymbol{k}) \leftarrow(\boldsymbol{i}, \boldsymbol{j}, \boldsymbol{k}) \backslash\left(i_{t}, j_{t}, k_{t}\right) ; \\
& \hat{\boldsymbol{u}} \leftarrow \arg \min _{\boldsymbol{u}}\left\|\mathcal{Y}-\sum_{t^{\prime}=1}^{t} u_{t^{\prime}} \mathcal{C}_{i_{t^{\prime}}, j_{t^{\prime}}, k_{t^{\prime}}}\right\|_{2} ; \\
& \mathcal{R} \leftarrow \mathcal{Y}-\sum_{t^{\prime}=1}^{t} \hat{u}_{t^{\prime}} \mathcal{C}_{i_{t^{\prime}}, j_{t^{\prime}}, k_{t^{\prime}}} ;
\end{aligned}
$$

for $t \leftarrow 1$ to $k$ do

$$
\left\lfloor\tilde{z}_{i_{t}, j_{t}, k_{t}} \leftarrow \hat{u}_{t}\right.
$$




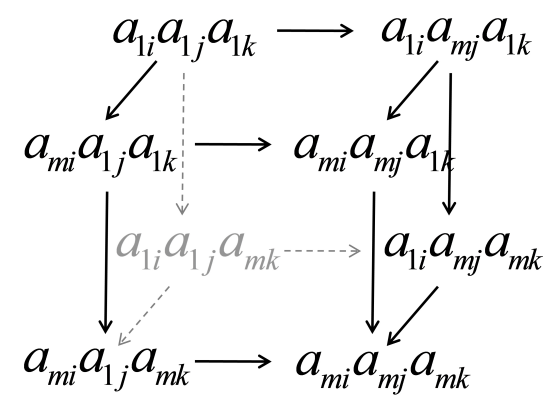

Figure 1: The structure of the 3D atom.

\subsection{D Projection}

We define the projection of the sample matrix $\mathcal{Y}$ onto $3 \mathrm{D} \mathcal{C}_{i, j, k}$ as:

$$
\frac{\left\langle\mathcal{Y}, \mathcal{C}_{i, j, k}\right\rangle}{\left\|\mathcal{C}_{i, j, k}\right\|_{2}}
$$

where $\left\langle\mathcal{Y}, \mathcal{C}_{i, j, k}\right\rangle \triangleq \mathcal{Y} \bullet_{1} \boldsymbol{a}_{i} \bullet_{2} \boldsymbol{a}_{j} \bullet_{3} \boldsymbol{a}_{k}$, and $\left\|\mathcal{C}_{i, j, k}\right\|_{2}$ is the Frobenius norm of $\mathcal{C}_{i, j, k}$, i.e.,

$$
\begin{aligned}
\left\|\mathcal{C}_{i, j, k}\right\|_{2} & \triangleq \sqrt{\sum_{i^{\prime}=1}^{m} \sum_{j^{\prime}=1}^{m} \sum_{k^{\prime}=1}^{m}\left(a_{i^{\prime} i} a_{j^{\prime} j} a_{k^{\prime} k}\right)^{2}} \\
& =\left\|\boldsymbol{a}_{i}\right\|_{2}\left\|\boldsymbol{a}_{j}\right\|_{2}\left\|\boldsymbol{a}_{k}\right\|_{2} .
\end{aligned}
$$

\subsection{Least Squares}

We construct an approximate to $\mathcal{Y}$ by $t$ atoms.

$$
\begin{aligned}
\tilde{\mathcal{Y}} & \approx \sum_{t^{\prime}=1}^{t} u_{i_{t^{\prime}}, j_{t^{\prime}}, k_{t^{\prime}}} \boldsymbol{c}_{i_{t^{\prime}}, j_{t^{\prime}}, k_{t^{\prime}}} \\
& \approx \sum_{t^{\prime}=1}^{t} u_{i_{t^{\prime}, j_{t^{\prime}}, k_{t^{\prime}}}} \boldsymbol{a}_{i_{t^{\prime}}} \otimes \boldsymbol{a}_{j_{t^{\prime}}}^{\mathrm{T}} \otimes \boldsymbol{a}_{k_{t^{\prime}}}^{\mathrm{T}} .
\end{aligned}
$$

A 3D matrix $\mathcal{R} \in \mathbb{R}^{m \times m \times m}$ is defined as the residue: $\mathcal{R}=\mathcal{Y}-\tilde{\mathcal{Y}}$. The optimization problem can be solved by finding the optimal $\boldsymbol{u}=\left(u_{i_{1}, j_{1}, k_{1}}, \cdots, u_{i_{t}, j_{t}, k_{t}}\right)^{\mathrm{T}}$ 
that minimizes the Frobenius norm of $\mathcal{R}$, which is in fact equivalent to the least square problem. Thus the optimization problem is equivalent to

$$
\hat{\boldsymbol{u}}=\arg \min _{\boldsymbol{u}}\|\mathcal{Y}-\tilde{\mathcal{Y}}\|_{2}^{2}
$$

According to (21) and $\operatorname{tr}\left((\cdot)^{\mathrm{T}}\right)=\operatorname{tr}(\cdot)$, we have

$$
\begin{aligned}
& \|\mathcal{Y}-\tilde{\mathcal{Y}}\|_{2}^{2} \\
= & \left\|\mathcal{Y}_{(1)}\right\|_{2}^{2}-2\left\langle\mathcal{Y}_{(1)}, \tilde{\mathcal{Y}}_{(1)}\right\rangle+\left\|\tilde{\mathcal{Y}}_{(1)}\right\|_{2}^{2} \\
= & \left\|\mathcal{Y}_{(1)}\right\|_{2}^{2}-2 \sum_{t^{\prime}=1}^{t} u_{i_{t^{\prime}}, j_{t^{\prime}}, k_{t^{\prime}}} \operatorname{tr}\left(\operatorname{vec}\left[\mathcal{C}_{i_{t^{\prime}}, j_{t^{\prime}}, k_{t^{\prime}}}\right] \mathcal{Y}_{(1)}^{\mathrm{T}}\right) \\
& +\sum_{t^{\prime}=1}^{t} \sum_{s^{\prime}=1}^{t} u_{i_{t^{\prime}}, j_{t^{\prime}}, k_{t^{\prime}}} u_{i_{s^{\prime}}, j_{s^{\prime}}, k_{s^{\prime}}} \cdot \operatorname{tr}\left(\operatorname{vec}\left[\mathcal{C}_{i_{t^{\prime}}, j_{t^{\prime}}, k_{t^{\prime}}}\right] \operatorname{vec}\left[\mathcal{C}_{i_{t^{\prime}}, j_{t^{\prime}}, k_{t^{\prime}}}\right]^{\mathrm{T}}\right),
\end{aligned}
$$

where the $\mathcal{Y}_{(1)}$ denotes the mode-n matricization of the tensor $\mathcal{Y}$ [20].

Let

$$
\mathbf{W}=\left(\begin{array}{ccc}
w_{1,1} & \cdots & w_{1, t} \\
\vdots & \vdots & \vdots \\
w_{t, 1} & \cdots & w_{t, t}
\end{array}\right)
$$

and

$$
\boldsymbol{v}=\left(\begin{array}{c}
\operatorname{tr}\left(\operatorname{vec}\left[\mathcal{C}_{i_{1}, j_{1}, k_{1}}\right] \mathcal{Y}_{(1)}^{\mathrm{T}}\right) \\
\vdots \\
\operatorname{tr}\left(\operatorname{vec}\left[\mathcal{C}_{i_{t}, j_{t}, k_{t}}\right] \mathcal{Y}_{(1)}^{\mathrm{T}}\right)
\end{array}\right)
$$

where

$$
w_{s, t}=\operatorname{tr}\left(\operatorname{vec}\left[\mathcal{C}_{i_{s}, j_{s}, k_{s}}\right] \operatorname{vec}\left[\mathcal{C}_{i_{t}, j_{t}, k_{t}}\right]^{\mathrm{T}}\right)
$$

Thus (23) can be rewritten as:

$$
\|\mathcal{Y}-\tilde{\mathcal{Y}}\|_{2}^{2}=\left\|\mathcal{Y}_{(1)}\right\|_{2}^{2}+\boldsymbol{u}^{\mathrm{T}} \mathbf{W} \boldsymbol{u}-2 \boldsymbol{u}^{\mathrm{T}} \boldsymbol{v}
$$


When $\|\mathcal{Y}-\tilde{\mathcal{Y}}\|_{2}^{2}$ takes the minimum, there must be

$$
\frac{\partial\|\mathcal{Y}-\tilde{\mathcal{Y}}\|_{2}^{2}}{\partial \boldsymbol{u}}=2 \mathbf{W} \boldsymbol{u}-2 \boldsymbol{v}=\mathbf{0}
$$

Hence

$$
\hat{\boldsymbol{u}}=\mathbf{W}^{-1} \boldsymbol{v}
$$

It is easy to get

$$
\operatorname{vec}\left[\mathcal{C}_{i_{s}, j_{s}, k_{s}}\right]=\boldsymbol{a}_{i_{s}} \otimes \boldsymbol{a}_{j_{s}} \otimes \boldsymbol{a}_{k_{s}}
$$

and

$$
\operatorname{vec}\left[\mathcal{C}_{i_{t}, j_{t}, k_{t}}\right]^{\mathrm{T}}=\boldsymbol{a}_{i_{t}}^{\mathrm{T}} \otimes \boldsymbol{a}_{j_{t}}^{\mathrm{T}} \otimes \boldsymbol{a}_{k_{t}}^{\mathrm{T}}
$$

Therefore,

$$
\begin{aligned}
w_{s, t} & =\operatorname{tr}\left(\operatorname{vec}\left[\mathcal{C}_{i_{s}, j_{s}, k_{s}}\right] \operatorname{vec}\left[\mathcal{C}_{i_{t}, j_{t}, k_{t}}\right]^{\mathrm{T}}\right) \\
& =\operatorname{tr}\left(\boldsymbol{a}_{i_{s}} \boldsymbol{a}_{i_{t}}^{\mathrm{T}}\right) \operatorname{tr}\left(\boldsymbol{a}_{j_{s}} \boldsymbol{a}_{j_{t}}^{\mathrm{T}}\right) \operatorname{tr}\left(\boldsymbol{a}_{k_{s}} \boldsymbol{a}_{k_{t}}^{\mathrm{T}}\right) \\
& =\left\langle\boldsymbol{a}_{i_{s}}, \boldsymbol{a}_{i_{t}}\right\rangle\left\langle\boldsymbol{a}_{j_{s}}, \boldsymbol{a}_{j_{t}}\right\rangle\left\langle\boldsymbol{a}_{k_{s}}, \boldsymbol{a}_{k_{t}}\right\rangle .
\end{aligned}
$$

\subsection{Matrix Inverse Update}

Obviously, $\mathbf{W}$ is a symmetric matrix which augments as the iteration increases. Hence we can compute its inverse matrix iteratively. Let $\mathbf{W}_{t-1}^{-1}$ be the inverse matrix of $\mathbf{W}_{t-1}$ at the $(t-1)$-th iteration. Then the matrix $\mathbf{W}_{t}$ can be represented as [21]:

$$
\mathbf{W}_{t}=\left(\begin{array}{cc}
\mathbf{W}_{t-1} & \boldsymbol{r}_{t} \\
\boldsymbol{r}_{t}^{\mathrm{T}} & \rho_{t}
\end{array}\right),
$$


where

$$
\rho_{t}=\left\langle\boldsymbol{a}_{j_{t}}, \boldsymbol{a}_{j_{t}}\right\rangle\left\langle\boldsymbol{a}_{i_{t}}, \boldsymbol{a}_{i_{t}}\right\rangle\left\langle\boldsymbol{a}_{k_{t}}, \boldsymbol{a}_{k_{t}}\right\rangle
$$

and

$$
\boldsymbol{r}_{t}=\left(\begin{array}{c}
\left\langle\boldsymbol{a}_{j_{t}}, \boldsymbol{a}_{j_{1}}\right\rangle\left\langle\boldsymbol{a}_{i_{t}}, \boldsymbol{a}_{i_{1}}\right\rangle\left\langle\boldsymbol{a}_{k_{1}}, \boldsymbol{a}_{k_{t}}\right\rangle \\
\vdots \\
\left\langle\boldsymbol{a}_{j_{t}}, \boldsymbol{a}_{j_{t-1}}\right\rangle\left\langle\boldsymbol{a}_{i_{t}}, \boldsymbol{a}_{i_{t-1}}\right\rangle\left\langle\boldsymbol{a}_{k_{t-1}}, \boldsymbol{a}_{k_{t}}\right\rangle
\end{array}\right)
$$

Thus,

$$
\mathbf{W}_{t}^{-1}=\left(\begin{array}{cc}
\mathbf{W}_{t-1}^{-1}+\frac{1}{\beta_{t}}\left(\boldsymbol{q}_{t} \boldsymbol{q}_{t}^{\mathrm{T}}\right) & \frac{1}{\beta_{t}} \boldsymbol{q}_{t} \\
\frac{1}{\beta_{t}} \boldsymbol{q}_{t}^{\mathrm{T}} & \frac{1}{\beta_{t}}
\end{array}\right)
$$

where

$$
\left\{\begin{array}{l}
\boldsymbol{q}_{t}=-\mathbf{W}_{t-1}^{-1} \boldsymbol{r}_{t} \\
\beta_{t}=\rho_{t}+\boldsymbol{r}_{t}^{\mathrm{T}} \boldsymbol{q}_{t}
\end{array}\right.
$$

Similarly, for $\boldsymbol{v}$, because

$$
\begin{aligned}
& \operatorname{tr}\left(\operatorname{vec}\left[\mathcal{C}_{i_{t}, j_{t}, k_{t}}\right] \mathcal{Y}_{(1)}^{\mathrm{T}}\right) \\
= & \operatorname{tr}\left\{\left(\boldsymbol{a}_{i_{t}} \otimes \boldsymbol{a}_{j_{t}}^{\mathrm{T}} \otimes \boldsymbol{a}_{k_{t}}^{\mathrm{T}}\right) \mathcal{Y}_{(1)}\right\} \\
= & \mathcal{Y} \bullet_{1} \boldsymbol{a}_{i_{t}} \bullet_{2} \boldsymbol{a}_{j_{t}} \bullet_{3} \boldsymbol{a}_{k_{t}},
\end{aligned}
$$

we have

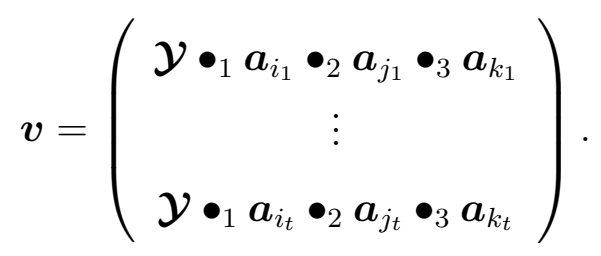




\section{Complexity and Memory Usage}

\subsection{Complexity}

\subsubsection{Project}

Let $\mathcal{P}$ be an $n \times n \times n$ matrix whose $(i, j, k)$-th element is $\left\|\mathcal{C}_{\boldsymbol{i , j , k}}\right\|_{2}$. Then this step can be implemented by $\mathcal{R} \times{ }_{1} \mathbf{A} \times{ }_{2} \mathbf{A} \times{ }_{3} \mathbf{A}$. $/ \mathcal{P}$. The complexity of this step is dominated by $(n \times m) \times(m \times m m)$ matrix-matrix multiplication, $(m n \times m) \times(m \times n)$ matrix-matrix multiplication, and $(n \times m) \times(m \times n n)$ matrix-matrix multiplication. As $m<n$, the complexity of this step is $O\left(m n^{3}\right)$.

\subsubsection{Select Best Matched Atoms}

Since there are $n^{3}$ atoms and $k \ll n^{3}$, the complexity of this step is approximately $O\left(n^{3}\right)$, negligible compared with the Project step.

\subsubsection{Renew Weights}

From (33), it can be seen that the complexity to calculate $\mathbf{W}_{t}$ is $O\left(m^{3}(t-\right.$ $1)$ ). From (36), the complexity of $\mathbf{W}_{t}^{-1}$ is $O\left((t-1)^{2}\right)$. The complexity to calculate $\boldsymbol{v}$ is dominated by $(1 \times m) \times(m \times m m)$ matrix-matrix multiplication, $(1 \times m) \times(m \times m)$ matrix-matrix multiplication, and $(1 \times m) \times(m \times 1)$ matrix-matrix multiplication. Thus its complexity is $O\left(\mathrm{tm}^{3}\right)$. Finally, the complexity to calculate the product of $\mathbf{W}_{t}^{-1}$ and $\boldsymbol{v}$ is $O\left(t^{2}\right)$.

\subsubsection{Update Residual}

The complexity of this step is decided by $t$. For $t \leq k \ll n^{3}$, the complexity of this step is negligible compared with the Project step. 
Based on the above analysis, we conclude that the complexity of the 3D-OMP is $O\left(m n^{3}\right)$.

\subsection{Memory Usage}

For the 3D-OMP, an $m \times n$ matrix is needed to accommodate the sampling matrix A. Hence the memory usage is $O(m n)$, which is the same as that of the 2D-OMP.

\section{Experimental Results}

In this section, we implement the 3D-OMP and KCS algorithms in MATLAB on Intel(R) Core(TM) i7 CPU with 12GB memory. Firstly, we perform an experiment to evaluate the performance of the 3D-OMP recovery algorith$\mathrm{m}$ for 3D $k$-sparse synthesized signals with the 3DSS. Secondly, we make the performance analysis of the 3D-OMP, KCS and standard CS. Meanwhile, we use a HSI to showcase the advantage of the 3D-OMP over the KCS algorithm. Finally, two HSIs are used to show the recovery quality.

\subsection{Perfect Reconstruction}

In the first experiment, we consider the synthesized 3D signals of size $16 \times 16 \times 16$ which are $k$-sparse in the 3D DCT domain. We test three typical settings $(n, k)=(16,4),(16,8)$, and $(16,16)$. For each $k$, we try 60 kinds of sample number $s=m \times m \times m$. For each $s$, we try $10^{3}$ trials. In each trial, we synthesize a $k$-sparse signal $\mathcal{Z}$ with normally distributed nonzero entries by using the sprandn function with density $k / n^{3}$, and generate the sampling matrix $\boldsymbol{\Phi}$ through sampling independent and identically distributed (i.i.d.) entries from standard Gaussian distribution. We evaluate the probability of 


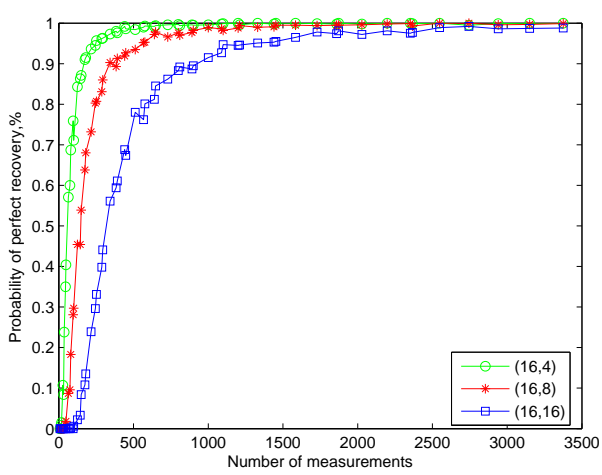

Figure 2: Perfect reconstruction of 3D-OMP.

perfect recovery (the signal estimated $\hat{\mathcal{Z}}$ obeys $\|\mathcal{Z}-\hat{\mathcal{Z}}\|_{2} \leq 10^{-5}$ ). The results are plotted in Fig. 2, which shows that fewer samples are needed to reconstruct the 3D sparse signals perfectly when the sparse level is small. As $k$ increases, more and more samples will be needed for perfect recovery. The sample-to-sparsity ratio $s / k$ for $90 \%$ perfect recovery are about 40,40 , and 51 for $k=4,8$, and 16 , respectively.

\subsection{Performance Analysis of 3D-OMP}

In this experiment we consider the synthesized 4-sparse 3D signals of size $8 \times 8 \times 8$ to compare the performance of the $3 \mathrm{D}$-OMP recovery algorithm with other three CS recovery schemes. The first scheme takes the 3D signal as a 1D signal, and recovers the signal from the global measurements using the standard recovery algorithm. The second scheme considers the $3 \mathrm{D}$ signal as a set of sections where a section is a 2D signal, and uses the KCS recovery from the set of measurements obtained independently from each $8 \times 8$ 1-section. The third scheme recovers each section independently from its individual measurements using the standard CS recovery algorithm. In our proposed 


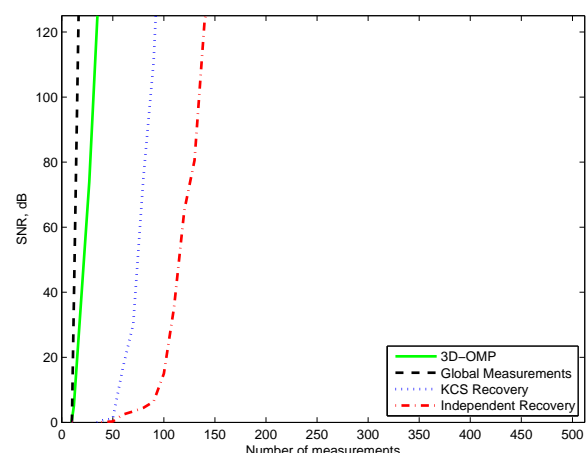

(a)

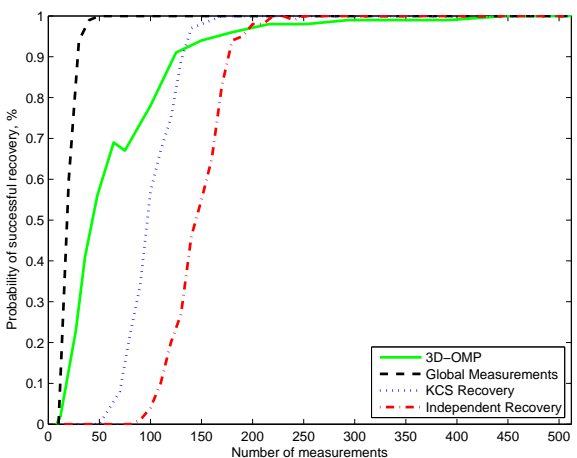

(b)

Figure 3: Performance analysis of 3D-OMP.

scheme, the 3D signal is sampled separately in the $x, y$ and $z$ dimensions, and recovered via the 3D-OMP algorithm. We try 18 kinds of measurement numbers $m$. For each value of $m$, we average $10^{2}$ iterations by generating 4-sparse signals $\mathcal{Z}$ with normally distributed nonzero entries with density $k / n^{3}$, and generate the sampling matrix $\boldsymbol{\Phi}$ through sampling independent and identically-distributed (i.i.d.) entries from standard Gaussian distribution. We evaluate the recovery signal-noise ratio (SNR) and probability of

success recovery (the signal estimated $\hat{\mathcal{Z}}$ obeys $\|\mathcal{Z}-\hat{\mathcal{Z}}\|_{2} \leq 10^{-3}\|\mathcal{Z}\|_{2}$ ). The empirical results are plotted in Fig. 3, which shows that the 3D-OMP recovery algorithm outperforms the KCS but provides lower success probabilities than the standard CS recovery from global measurements.

\subsection{Algorithm Comparison}

We compare the performance of the 3D-OMP with that of the KCS using a real-world hyperspectral image of size $N=128 \times 128 \times 16$. The $M$ denotes the number of measurements. Firstly, we sparsify the 3D signal with the 


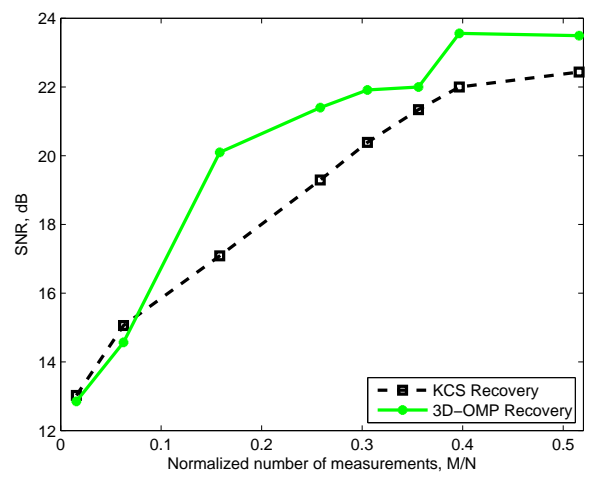

(a)

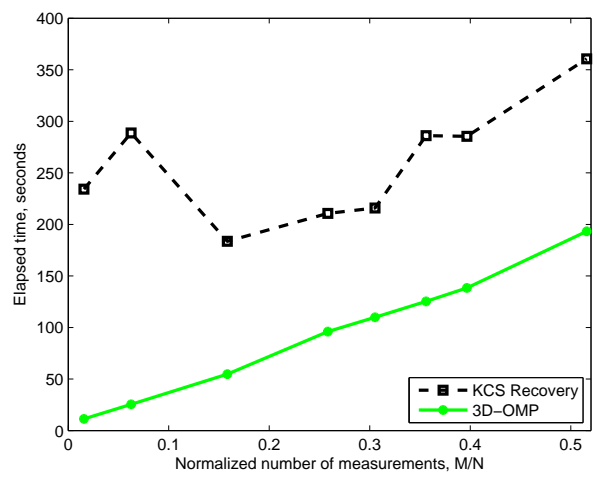

(b)

Figure 4: Comparison of 3D-OMP with KCS.

transform base $\boldsymbol{\Psi}_{1}$ for the spatial dimension and $\boldsymbol{\Psi}_{2}$ for the spectral dimension where the bases are the Karhunen-Loeve transform (KLT) matrix. Then we sample the $3 \mathrm{D}$ signals with $\boldsymbol{\Phi}_{1}$ for the horizontal and vertical dimensions and $\boldsymbol{\Phi}_{2}$ for the spectral dimension where the sampling matrices are formed by i.i.d. entries from standard Gaussian distribution. We test 8 kinds of measurements for both algorithms and then plot the SNR and elapsed time in Fig. 4 (a) and Fig. 4(b), respectively. It is seen that when the measurement number is small the proposed 3D-OMP algorithm achieves higher recovery quality and elapses less time. As the number of measurements increases, the advantage of the 3D-OMP over the KCS vanishes gradually. This is because the complexity of the 3D-OMP is dominated by the Project step. The complexity will ascend when the sample number increases, which explains the above phenomenon. 


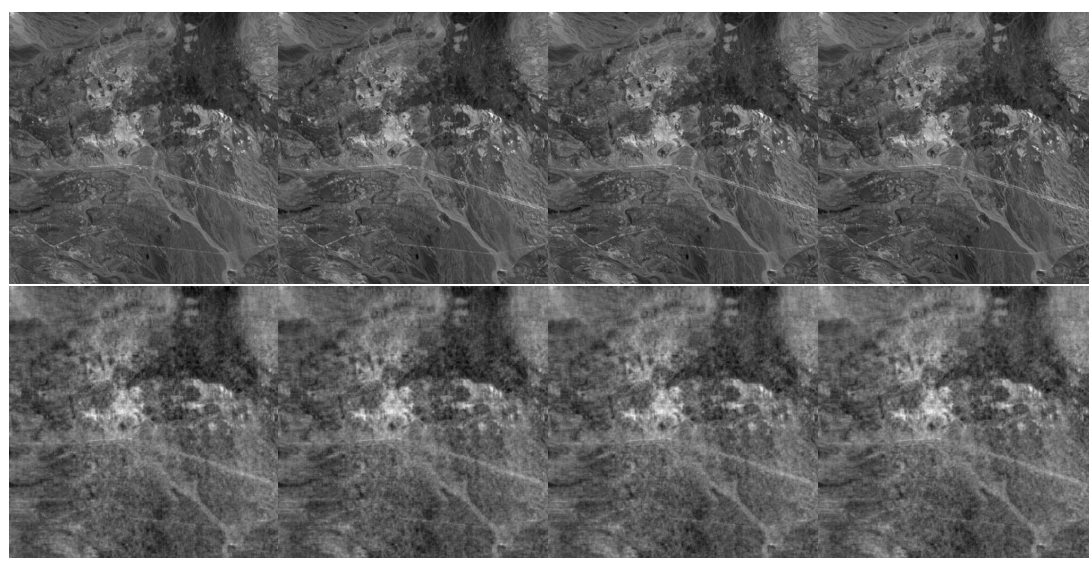

(a)

(b)

(c)

(d)

Figure 5: Recovered cuprite images by 3D-OMP, where the compression ratio is 8 and the SNR is $22.45 \mathrm{~dB}$. (a) the original image of the 1st band (top) and its recovered image (bottom), (b) the original image of the 3th band (top) and its recovered image (bottom), (c) the original image of the 5th band (top) and its recovered image (bottom), (d) the original image of the 9 th band (top) and its recovered image (bottom). 


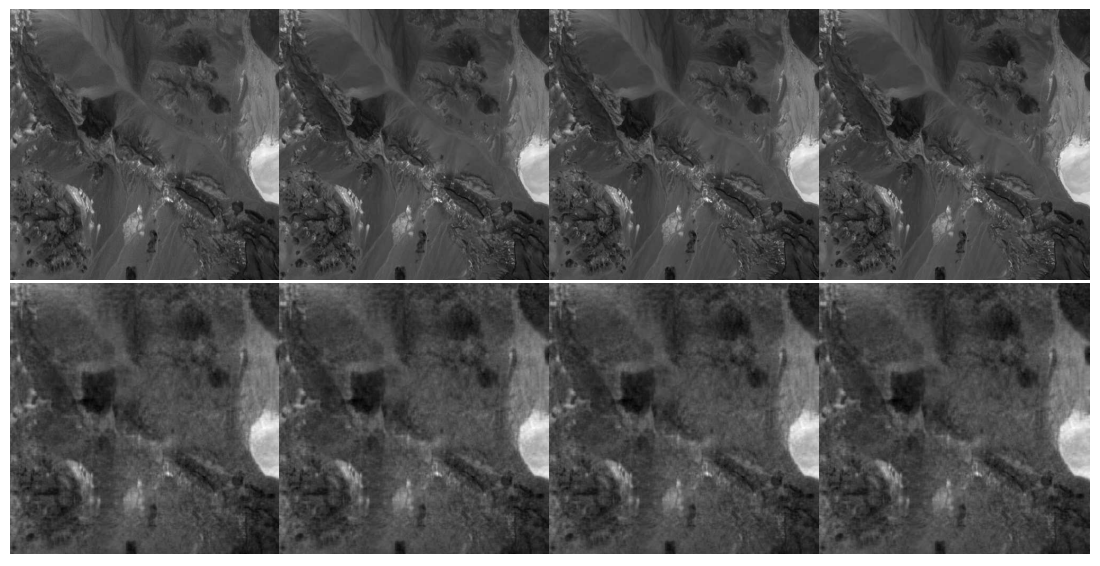

(a)

(b)

(c)

(d)

Figure 6: Recovered lunar images by 3D-OMP, where the compression ratio is 8 and the SNR is $23.41 \mathrm{~dB}$. (a) the original image of the 1st band (top) and its recovered image (bottom), (b) the original image of the 3th band (top) and its recovered image (bottom), (c) the original image of the 5th band (top) and its recovered image (bottom), (d) the original image of the 9 th band (top) and its recovered image (bottom). 


\subsection{Recovery Quality}

In this subsection, we have tested two HSIs of size $512 \times 512 \times 16$ to show the recovery quality of the 3D-OMP algorithm. The sparsifying transform matrices are the KLT matrix $\boldsymbol{\Psi}_{h}$ for the spatial domain and $\boldsymbol{\Psi}_{v}$ for the spectral domain. We sample the signal with $\boldsymbol{\Phi}_{h}$ in the spatial dimension and $\Phi_{v}$ in the spectral dimension, in which the i.i.d. entries are drawn from the standard Gaussian distribution. The compression ratio is 8. As the realworld images do not satisfy $k$-sparse $\left(k \ll n^{3}\right)$ constraint in some sparsity domain very well, $k$ is selected based on the practical HSI. For both the cuprite and lunar images, the sparse level $k$ are set to 1500 , meaning that 1500 spikes are recovered. The original images of the 1st, 3th, 5th and 9th bands and their recovered images are shown in Fig. [5and Fig. 6, respectively, where the SNR of cuprite is $22.45 \mathrm{~dB}$ and the SNR of lunar is $23.41 \mathrm{~dB}$. The latter is better.

\section{Conclusion}

In this paper, we have devised the 3D-OMP recovery algorithm. Moreover, we provide the performance analysis for the proposed recovery approach. The experimental results demonstrate that the 3D compressive scheme can use fewer samples and shorter time to reconstruct the 3D signal. Compared with the KCS recovery scheme, our proposal reduces the recovery complexity significantly and maintains high recovery quality. Hence, the proposed 3D-OMP algorithm is a promising solution for HSI applications. 


\section{Acknowledgments}

This work was supported by the National Science Foundation of China (No. 61271280 and 61001100), Provincial Science Foundation of Shaanxi,

China (No. 2010K06-15) and Provincial Scientific Research and Development Plan of Shaanxi, China (No. 2015NY049).

\section{References}

[1] M. F. Duarte and R. G. Baraniuk, "Kronecker compressive sensing," IEEE Transaction on Image Processing, vol. 21, no. 2, pp. 494-504, Feb. 2012.

[2] E. Candes, J. Romberg, and T. Tao, "Robust uncertainty principles: Exact signal reconstruction from highly incomplete frequency information," IEEE Transaction on Information Theory, vol. 52, no. 2, pp. 489-509, Feb. 2006.

[3] E. Candes and T. Tao, "Near optimal signal recovery from random projections: Universal encoding strategies," IEEE Transaction on Information Theory, vol. 52, no. 12, pp. 5406-5425, Dec. 2006.

[4] D. Donoho, "Compressed sensing," IEEE Transaction on Information Theory, vol. 52, no. 4, pp. 1289-1306, April. 2006.

[5] D. Needell and R. Vershynin, "Signal Recovery From Incomplete and Inaccurate Measurements via Regularized Orthogonal Matching Pursuit," IEEE Journal of Selected Topics in Signal Processing, vol. 4, no. 2, pp. 310-316, April. 2010. 
[6] D. Needell and J. A. Tropp, "CoSaMP: Iterative signal recovery from incomplete and inaccurate samples," Applied and Computational Harmonic Analysis, vol. 26, no. 3, pp. 301-321, May, 2009.

[7] W. Dai and O. Milenkovic, "Subspace Pursuit for Compressive Sensing Signal Reconstruction," IEEE Transaction on Information Theory, vol. 55, no. 5, pp. 2230-2249, May. 2009.

[8] J. Tropp and A. Gilbert, "Signal recovery from random measurements via orthogonal matching pursuit," IEEE Transaction on Information Theory, vol. 53, no. 12, pp. 4655-4666, Dec. 2007.

[9] G. Pope, "Compressive sensing: a summary of reconstruction algorithms," Master Thesis. Zürich: Eidgenössische Technische Hochschule. 2009

[10] Y. Rivenson and A. Stern, "Compressed imaging with a separable sensing operator," IEEE Signal Process. Lett., vol. 16, no. 6, pp. 449-452, June. 2009.

[11] Y. Fang, J. J. Wu, and B. Huang, "2D sparse signal recovery via 2D orthogonal matching pursuit," Sci China Inf Sci, vol. 55, no. 4, pp. 889-897, April. 2012.

[12] V. Stanković, L. Stanković, and S. Cheng, "Compressive video sampling," in EUSIPCO, Lausanne, Switzerland, pp. 25-29, Aug. 2008.

[13] J. Zheng and E. L. Jacobs, "Video compressive sensing using spatial domain sparsity," SPIE Optical Engineering, vol. 48, no. 8, Aug. 2009. 
[14] L. Kang and C. Lu, "Distributed compressive video sensing," in ICASSP, pp. 1169-1172, Taipei, China, April. 2009.

[15] M. Golbabaee and P. Vandergheynst, "Hyperspectral images compressed sensing via low-rank and joint-sparse matrix recovery," in ICASSP, pp. 2741-2744, Lausanne, Switzerland, March. 2012.

[16] X. B. Shu and N. Ahuja, "Imaging via three-dimensional compressive sampling (3DCS)," in ICCV, pp. 439-446, Barcelona, Spain, Nov. 2012.

[17] P. Ye, J. L. Paredes, and Y. Wu, et al, "Compressive confocal microscopy: 3D reconstruction algorithm," Proc. of SPIE, Emerging Digital Micromirror Device Based Systems and Applications, vol. 7210, San Jose, Feb, 13. 2009.

[18] Chengbo Li, Ting Sun, Kevin Kelly, and Yin Zhang, "A Compressive Sensing and Unmixing Scheme for Hyperspectral Data Processing," IEEE Transaction on Image Processing, vol. 21, no. 3, pp. 1200-1201, Feb. 2012.

[19] Horn R A, Johnson C R. "Topics in Matrix Analysis," Cambridge: Cambridge University Press, 1991

[20] Tamara G. Kolda, Brett W. Bader. "Tensor Decompositions and Application," SIAM Rev., vol. 51, no. 3, pp. 455-500, 2009

[21] B. Noble and J. W. Danniel, "Applied Linear Algebra. 3rd ed," Englewood Cliffs, NJ: Prentice-Hall, 1988 


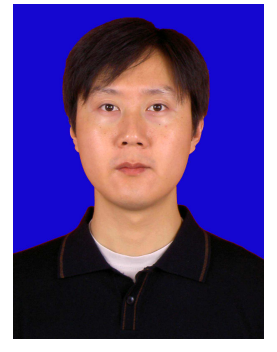

Yingqiu Huo received his B.Eng. in computer science and technology, M.Eng., and Ph.D degrees in agricultural electrification and automation from Northwest A\&F University Yangling, China, in 2003, 2006 and 2015 respectively. He is currently a Experimentalist with Northwest A\&F University. His research interests are multi-dimensional signal processing and parallel computing.

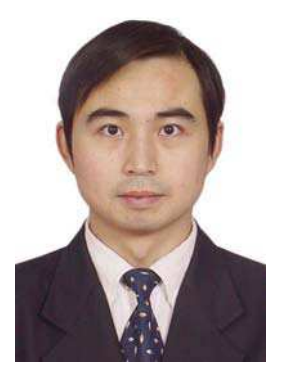

Yong Fang received his B.Eng., M.Eng., and Ph.D. degrees from Xidian University, Xian, China, in 2000, 2003 and 2005, respectively, all in signal processing. In 2005, he was appointed as a Post-Doctoral Fellow for one year with Northwest Polytechnical University, Xian. From 2007 to 2008, he was with Hanyang University, Seoul, Korea, as a Research Professor. He is currently a full Professor with Northwest A\&F University, Yangling, China. He has long experiences in hardware development, e.g., FPGA-based video codec design, DSP-based video surveillance systems, and so on. His current research interests include distributed source coding, image/video coding, processing, and transmission.

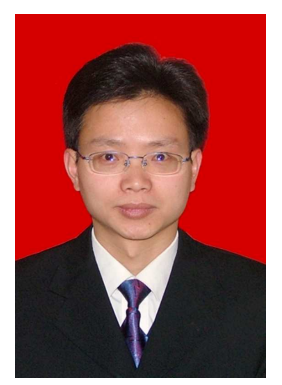

Lei Huang received the B.Sc., M.Sc., and Ph.D. degrees in electronic engineering from Xidian University, Xian, China, in 2000, 2003, and 2005, respectively. From 2005 to 2006, he was a Research Associate with the Department of Electrical and Computer Engineering, Duke University, Durham, NC. From 2009 to 2010, he was a Research Fellow with the Department of Electronic Engineering, City University of Hong Kong and a Research Associate 
with the Department of Electronic Engineering, The Chinese University of Hong Kong. Since 2011, he has joined the Department of Electronic and Information Engineering, Harbin Institute of Technology Shenzhen Graduate School, where he is currently a Professor. His research interests include spectral estimation, array signal processing, statistical signal processing, and their applications in radar and wireless communication systems. He currently is an editorial board member of Digital Signal Processing. 


\section{Abstract}

Though many three-dimensional (3D) compressive sensing schemes have been proposed, recovery algorithms in most of these schemes are designed for $1 \mathrm{D}$ or $2 \mathrm{D}$ signals, which cause some serious drawbacks, e.g., huge memory usage, and high decoder complexity. This paper proposes a 3D separable operator (3DSO) which is able to completely exploit the spatial and spectral correlation to sparsify and samples the 3D signal in three dimensions. A 3D orthogonal matching pursuit (3D-OMP) algorithm is then employed to recover the 3D sparse signal, which is able to reduce the computational complexity of the decoder significantly with guaranteed accuracy. In the proposed algorithm, we represent each 3D signal as a weighted sum of 3D atoms, which allow us to sample the 3D signal with 3D separable sensing operator. Then the best matched atoms are selected to construct the 3D support set, and the 3D signal is optimally recovered from the 3D support set in the sense of the least squares.

We have performed some experiments to evaluate the performance of the 3D-OMP, KCS, global measurements and independent recovery. Experimental results show that the 3D-OMP approach achieves better recovery quality and gains higher probability of successful recovery than the KCS.

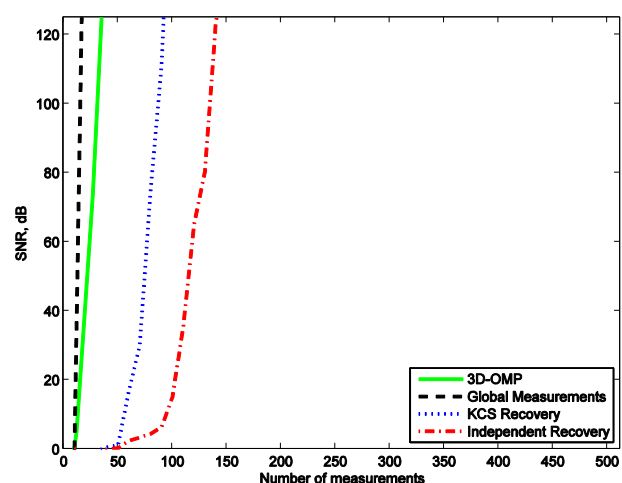

(a)

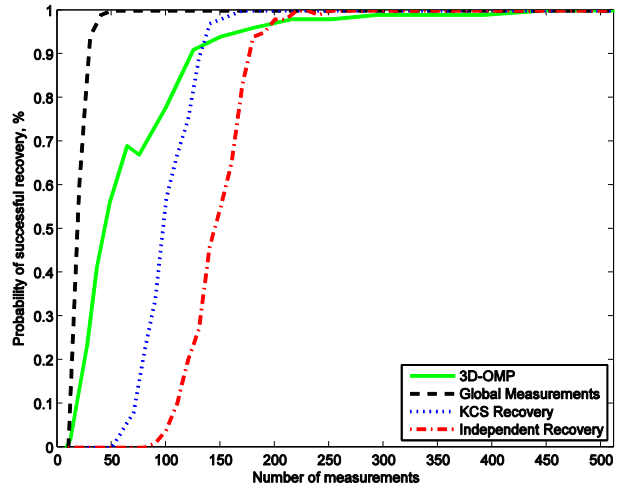

(b)

Figure 3: Performance analysis of 3D-OMP. 